Article

\title{
Associations between Alternate Healthy Eating Index-2010, Body Composition, Osteoarthritis Severity, and Interleukin-6 in Older Overweight and Obese African American Females with Self-Reported Osteoarthritis
}

\author{
Macy Mears ${ }^{1}$, Lisa Tussing-Humphreys ${ }^{2,3,4}$, Leah Cerwinske ${ }^{1}$, Christy Tangney $^{1}{ }^{1}$, \\ Susan L. Hughes ${ }^{2,5}$, Marian Fitzgibbons ${ }^{2,3}$ and Sandra Gomez-Perez ${ }^{1, *}$ \\ 1 Department of Clinical Nutrition, College of Health Sciences, Rush University, Chicago, IL 60601, USA; \\ mearsmacy@gmail.com (M.M.); Leah_A_Cerwinske@rush.edu (L.C.); Christy_Tangney@rush.edu (C.T.) \\ 2 Institute for Health Research and Policy, University of Illinois at Chicago, Chicago, IL 60601, USA; \\ ltussing@uic.edu (L.T.-H.); shughes@uic.edu (S.L.H.); mlf@uic.edu (M.F.) \\ 3 University of Illinois Cancer Center, University of Illinois at Chicago, Chicago, IL 60601, USA \\ 4 Department of Medicine, College of Medicine, University of Illinois at Chicago, Chicago, IL 60601, USA \\ 5 School of Public Health, University of Illinois at Chicago, Chicago, IL 60601, USA \\ * Correspondence: sandra_1_gomez-perez@rush.edu
}

Received: 8 November 2018; Accepted: 18 December 2018; Published: 22 December 2018

check for updates

\begin{abstract}
Osteoarthritis (OA) is a leading cause of immobility in the United States and is associated with older age, inflammation, and obesity. Prudent dietary patterns have been associated with disease prevention, yet little evidence exists describing diet quality (DQ) in older overweight or obese African American (AA) adults with OA and its relation to body composition. We conducted a secondary data analysis of a dataset containing alternate Healthy Eating Index-2010 (AHEI-2010), body composition, OA severity, and serum interleukin-6 (IL-6) data from 126 AA females (aged 60-87 years) with OA to examine the relationships between these variables. Our sample had poor DQ and reported having higher OA severity as measured by the Western Ontario and McMaster Universities Osteoarthritis Index (WOMAC). Interleukin-6 was negatively correlated with AHEI-2010, and AHEI-2010 and the WOMAC physical function subcategory (WOMACpf) were significant predictors of IL-6 (odds ratio (OR): 0.95 , 95\% confidence interval (CI) $0.92-0.99$ and 1.04, 95\% CI 1.01-1.07, respectively, $p<0.05$ ) but not body composition. In conclusion, AHEI-2010 and WOMACpf were significant predictors of inflammation (IL-6) and AHEI-2010 accounted for 16\% of the variation of IL-6 (inflammation) in this sample.
\end{abstract}

Keywords: AHEI-2010; body composition; inflammation; osteoarthritis; obesity; African Americans; older adults

\section{Introduction}

Osteoarthritis (OA), primarily affecting the hip, knee, and hand joints, is the most common type of arthritis with $\sim 40 \%$ of adults over the age of 40 having some degree of knee OA [1]. OA is characterized by a breakdown of articular cartilage and low-grade inflammation [2]. It is estimated that 1 in 5 adults in the United States will be 65 years or older by 2030 [3], giving rise to an older population living longer with chronic diseases and ailments such as OA [4]. Maintaining quality of life and health in aging is important and modifiable factors, like diet and exercise, play important roles [5]. Using the National Health and Nutrition Examination Survey (NHANES) III, oversampled for minorities and 
specific to those 60 years and older, $47.3 \%$ of all individuals ( $50.5 \%$ women and $43.1 \%$ men) had at least some degree of radiographic evidence of knee OA [6]. Higher radiographic knee OA is reported for African Americans (AA) compared to Mexican-Americans or Non-Hispanic Whites (NHW) $(52.4 \%$, $39.7 \%$, and $36.2 \%$, respectively, $p<0.01$ ) and the highest prevalence of $\mathrm{OA}$ is reported specifically among AA women (60.2\%, 95\% CI: 52.8-67.5\%) [6].

OA is one of the leading causes of immobility in older adults living in the U.S. and is often closely associated and/or exacerbated by obesity [7]. Findings from a meta-analysis of 47 observational studies showed that preventing obesity, often a low-grade inflammatory state [8,9], could reduce the risk of knee OA by up to $50 \%$ [10]. Additionally, overweight was associated with two-times higher odds of OA while obesity had almost four times higher odds of OA compared to adults with normal body mass index (BMI) [10]. OA symptoms, including chronic pain, morning stiffness, and sensations of grating joints may also be related to systemic and local (synovial) low-grade inflammation [11,12]. The Western Ontario and McMaster Universities Osteoarthritis Index (WOMAC) score captures the degree of OA symptom severity and has been validated as a self-administered health status measure in adults with hip or knee OA [13]. Similar to weight gain, obesity-driven changes to body fat accumulation and distribution, such as increased proportions of abdominal fat tissue, are also postulated as major contributing factors of low-grade inflammation [14]. Of particular interest, excess visceral adipose tissue (VAT), located in the intra-abdominal region, is consistently associated with inflammation and chronic disease development; however, the exact link between VAT and OA development is unknown [14]. Taken together, the evidence suggests that obesity in some older adults, primarily in the intra-abdominal region, is an important driver of inflammation and OA.

High adherence to dietary patterns such as, the Harvard Healthy Eating Plate, which encourages a diet rich in fruits, vegetables, fish and whole grains, with moderate consumption of alcohol and low intake of sugar-sweetened beverages, is associated with disease prevention and longevity [15]. Quantifying adherence to a given dietary pattern using statistical methods or a scoring index, such as the alternate Healthy Eating Index (AHEI) which assesses adherence to the Harvard Health Eating Plate, allows examination of associations between diet quality (DQ) and health [16].

Little to no evidence exists exploring links between OA, obesity, and dietary patterns and much less in older AA females. Additionally, it is unclear if better DQ has beneficial effects on body composition, such as regional fat distribution defined by VAT or overall body fat percent (BF\%) or if it is associated with lower systemic inflammation and lower OA severity, particularly for older AAs with OA. Thus, the purpose of this study was to examine the associations between DQ, measured by the AHEI-2010, body composition, serum interleukin-6 (a biomarker of inflammation), and OA severity in older AA adults with self-reported OA.

\section{Materials and Methods}

\subsection{Study Design}

This study was a cross-sectional analysis of data collected at baseline from a subset of subjects accrued to the Fit \& Strong! Plus comparative effectiveness trial, herein referred to as the parent study (\#R01AG039374) [17,18]. A full description of the parent study design and methods have been published elsewhere [17].

An ancillary study to the parent study was conducted (American Cancer Society of Illinois, \#2617755), to explore changes in circulating biomarkers and body composition from baseline to post-intervention in a subset of subjects [18]. Data collected as part of the parent and ancillary studies served as the basis for this analysis to examine relationships between DQ (AHEI-2010), circulating inflammatory marker (IL-6), body fat composition, including BF\% and VAT, and OA severity in older AA adults with self-reported OA who were overweight or obese.

Briefly, participants were overweight and obese older adults with self-reported OA of the knee or hip, referred to as lower extremity (LE) OA who resided in the Chicagoland area [17]. The ancillary 
study specifically targeted the AA participants from the first eight iterations of the parent trial. Eligibility criteria including: 60 years or older, overweight or obese (BMI $25-50 \mathrm{~kg} / \mathrm{m}^{2}$ ), not meeting physical activity guidelines of at least $150 \mathrm{~min}$ per week, able to attend interviews and weekly classes, and self-reported LE OA (defined as acknowledging knee or hip region pain most days in the last month or remembering knee, hip, lower back, ankles, or feet pain most days of at least one month in the past six months). Exclusion criteria included: rheumatoid arthritis or other health conditions that contraindicated exercise, uncontrolled diabetes, hip/knee surgery in the past six months, intention for hip/knee surgery within the upcoming year, steroid injections in the previous three months in hip/knee regions, or a score of one or more on an exercise and high-risk screener [17]. Additionally, the ancillary study included individuals who identified as AA, were able to attend University of Illinois at Chicago (UIC) for visits, and were willing to undergo fasting blood tests and dual energy X-ray absorptiometry (DXA) scans. It excluded subjects who had cancer within the past five years, a body weight of $>450$ pounds $(204.5 \mathrm{~kg}$ ), or those unwilling to refrain from medications that could affect blood biomarker results [18]. All participants provided written informed consent. This secondary analysis using de-identified data was exempt from full Institutional Review Board submission under Policy RA-IRB-118 for non-human subjects research at Rush University.

\subsection{Demographics, Subject Characteristics, and Anthropometrics}

At baseline of the parent study, trained research staff collected data in person with a sociodemographic questionnaire (e.g., age, socioeconomic status, education). A medical health history questionnaire to ascertain information about major comorbidities (e.g., diabetes, hypertension, cardiovascular disease) was administered. Height was measured with a portable stadiometer (Seca, Chino, CA) to the nearest $0.5 \mathrm{~cm}$ in duplicate following the standard. Body weight was measured using a commercial digital scale (Tanita BWB 800, Arlington Heights, IL, USA) to the nearest $0.1 \mathrm{~km}$ in duplicate following standard procedures. Body mass index (BMI) was calculated as weight in kilograms divided by height in meters squared $\left(\mathrm{kg} / \mathrm{m}^{2}\right)$. A BMI value $25-29.9 \mathrm{~kg} / \mathrm{m}^{2}$ was considered overweight and $\geq 30 \mathrm{~kg} / \mathrm{m}^{2}$ obese [19].

\subsection{Severity of Osteoarthritis $(O A)$}

The WOMAC questionnaire, a validated tool in various populations, which is used to measure severity of OA, assigns total scores between 0-96 points based on three separate components of OA severity: physical function (0-68 points), stiffness (0-8 points), and pain ( $0-20$ points) [13]. For this study, total WOMAC scores and the subcomponent scores for physical function (WOMACpf) were analyzed. Higher total WOMAC scores represent worse OA severity, and higher physical function subcomponent score, WOMACpf, $(>20)$ indicate worse physical function. The sample was dichotomized based on highest (scores $>20$ ) versus lowest (scores $\leq 20$ ) WOMACpf subcategory scores (i.e., higher scores are indicative of worse physical function) to explore if there were differences in DQ and/or inflammation between these two severity groups.

\subsection{Body Composition}

Body composition estimates of $\mathrm{BF} \%$ and VAT were obtained via whole body DXA scan (GE Healthcare iLunar DXA, WI, USA). Subjects were required to fast $8 \mathrm{~h}$ prior to the scan, abstain from exercise, and wear light clothing with prohibited items removed from pockets on the day of scan as previously described [17]. A half-body scan, following manufacturer specifications [18], was conducted on participants whose body fell outside of the scan's field of view.

\subsection{Circulating Inflammatory Marker}

Fasting antecubital venous blood samples were collected by trained phlebotomists and processed for serum following standard methods. IL-6 was assessed using a high sensitivity (hs) enzyme-linked 
immunosorbent assay (ELISA) (R\&D Systems, Minneapolis, MN, USA). Average coefficient of variation for hs-IL-6 was $9.18 \%$.

\subsection{Diet Quality}

Dietary intake was quantified from an interviewer-administered Block 2005 Food Frequency Questionnaires (FFQ) (NutritionQuest, Berkeley, CA, USA) [20-22]. The FFQ consisted of 110 questions (food items and supplements). Block 2005 FFQs were sent to NutritionQuest (Berkeley, CA, USA) for analysis including estimates for AHEI-2010. FFQs with total energy intake of $<500$ and $>3500$ calories (kcals) per day for females were deemed implausible [23,24]. The original data contained 134 females, but after adjusting for plausible caloric intake, final analysis included 126 females. Details for calculating AHEI-2010 have been published elsewhere [25]. The AHEI-2010 scores can range from 0 to 110 points with higher scores reflecting greater adherence to the Harvard Healthy Eating Plate [25].

\subsection{Statistical Analysis}

The statistical program used was IBM SPSS Statistics for Windows, Version 22 Premium (IBM Corp, Armonk, NY, USA). Continuous variables, including age, total WOMAC score, BMI, BF\%, VAT, AHEI-2010, and IL-6, were described using median and interquartile ranges (IQR) or mean \pm standard deviation (SD) based on normality. Spearman and partial correlational coefficients were conducted to determine associations between AHEI and body composition (VAT and BF\%) and AHEI-2010 and inflammation (IL-6). For variables with significant $p$-values, the magnitude of the association was determined using an effect size chart with the coefficient of determination $\left(r^{2}\right)$ [26]. WOMACpf subcategory, a subcomponent of the overall WOMAC score, was identified as a potential confounder between DQ and inflammation in the sample and included in the models as a covariate. Logistic regression models were conducted to assess the relationship between DQ and inflammation, adjusted for $\mathrm{BF} \%$ or VAT with high or low outcome variables for IL-6 (median splits based on $\geq 3.5 \mathrm{pg} / \mathrm{mL}$ for hi and $<3.5 \mathrm{pg} / \mathrm{mL}$ for lo) with low as the reference category. To assess the strength of the relationship between predictors and the prediction, as well as the predictability of logistic regression models, Cox and Snell R squared and Nagelkerke R squared statistics were used. Significance was set at a $p$-value of $<0.05$.

\section{Results}

\subsection{Sample Characteristics}

A total of 126 AA females were analyzed. Demographic and clinical characteristic data are presented in Table 1. The majority of females attended some or at least four years of college. Average BMI was $35.2 \pm 5.8 \mathrm{~kg} / \mathrm{m}^{2}$. Over $50 \%$ of females had BMI $\geq 35 \mathrm{~kg} / \mathrm{m}^{2}$, indicative of higher severity of obesity in this older adult group. The median BF\% was approximately $47 \%$ and the median VAT volume was $1494 \mathrm{~cm}^{3}$. About $56 \%$ of the sample scored higher WOMAC scores indicating higher or worse severity of OA.

Approximately $44 \%$ of the sample (Table 1 ) reported higher WOMACpf scores (i.e., higher scores are indicative of worse physical function). Age, comorbidity distribution, and BF\% did not significantly differ between groups. However, those with higher BMI $\left(\geq 35 \mathrm{~kg} / \mathrm{m}^{2}\right)$ reported worse physical function. Median VAT volume was greater for those with higher scores $\left(1703 \mathrm{~cm}^{3}\right.$ vs. $1309 \mathrm{~cm}^{3}$ respectively, $p=0.002)$. IL-6 was significantly higher for women with higher WOMAC scores versus the lower score group, $4.1 \mathrm{pg} / \mathrm{mL}(0.1,19.8)$ vs. $3.3 \mathrm{pg} / \mathrm{mL}(0.7,16.3)$, respectively $(p=0.040)$. There were no significant differences of AHEI-2010 scores between females with high versus low WOMAC scores $(55.8 \pm 10.2$ vs. $58.5 \pm 11.2, p=0.171)$. 
Table 1. Demographic and clinical characteristics of sample.

\begin{tabular}{lc}
\hline \multicolumn{1}{c}{ Variables } & Females $(\boldsymbol{n}=\mathbf{1 2 6})$ \\
\hline Age, years, median (IQR) & $65(60,87)$ \\
\hline Education Level, $n(\%)$ & $0(0)$ \\
None or kindergarten & $1(0.8)$ \\
Grades 1-8 & $6(4.8)$ \\
Grades 9-11 & $16(12.7)$ \\
Grade 12 or GED & $54(42.9)$ \\
College 1-3 years or tech school & $49(38.9)$ \\
College 4 years or more & $24(1,86)$ \\
\hline WOMAC Total Score, 0-96 points, median (IQR) & \\
\hline WOMACpf Score, $n(\%)$ & $70(55.6)$ \\
0-19 points & $56(44.4)$ \\
20-68 points & \\
\hline Current Health Insurance Coverage ${ }^{\mathrm{a}}, n(\%)$ & $17(13.5)$ \\
Medicare A & $58(46)$ \\
Medicare A \& B & $19(15.1)$ \\
Medicaid & $17(13.5)$ \\
Medicare HMO & $55(43.7)$ \\
Private/Supplemental & \\
\hline Comorbidities ${ }^{b}$, yes, $n(\%)$ & $8(6.3)$ \\
Diabetes & $6(4.8)$ \\
Stroke/Paralysis & $2(1.6)$ \\
Heart Disease & $30(23.8)$ \\
High Blood Pressure & $18(14.3)$ \\
Canh Cholesterol & $5(4)$ \\
\hline Body Mass Index, kg/m ${ }^{2}$, mean \pm SD & $35.2 \pm 5.8$ \\
BMI Category, $n(\%)$ & $62(49.2)$ \\
$<35$ kg/m ${ }^{2}$ & $64(50.8)$ \\
\hline Body kg/m ${ }^{2}$ & $46.7(30.2,57.2)$ \\
\hline Visceral Adipose Tissue volume, $\left(\mathrm{cm}{ }^{3}\right)$, median $(\mathrm{IQR})$ & $1494(53,3504)$ \\
\hline Interleukin-6, pg/mL, median $(\mathrm{IQR})$ & $0.1,19.8)$ \\
\hline
\end{tabular}

IQR: interquartile range, GED: general education development, WOMAC: Western Ontario and McMaster Universities Osteoarthritis Index, used to determine severity of osteoarthritis (OA) symptoms (96 points = worst severity), WOMACpf: Western Ontario and McMaster Universities Osteoarthritis Index physical function category (20-68 points = worse physical function), HMO: health maintenance organization, BMI: body mass index. ${ }^{\text {a }}$ Missing data for current health insurance coverage for 5 females ( $4 \%){ }^{\text {b }}$ Missing data for comorbidities for 91 females $(72.2 \%)$.

\subsection{Nutrient Intakes and Diet Quality as Measured by Alternate Healthy Eating Index-2010 (AHEI-2010)}

Median (interquartile range, IQR) total daily caloric intake of the sample and selected median estimates of dietary nutrients are shown in Table 2. The median overall AHEI-2010 score was $57.4 \pm 10.9$ points (Table 3). The AHEI-2010 score for median servings of total fruit (not including juice) was low at $3.1(0.1,10)$, as well as the score (reverse scored) for sugar-sweetened beverages and fruit juices $(1.04(0,10))$, meaning the majority of the females studied consume these beverages daily. No differences in mean AHEI-2010 scores were observed between those females with lower vs. higher WOMAC scores (58.5 \pm 11.2 vs. $55.8 \pm 10.2, p=0.171)$. 
Table 2. Nutrient intake data of females.

\begin{tabular}{lc}
\hline \multicolumn{1}{c}{ Variables } & Females $(\boldsymbol{n}=\mathbf{1 2 6})$ \\
\hline Total Daily Calories, kcals & $1389(892)$ \\
\% daily kcals from carbohydrates & $46.9 \pm 8.8$ \\
\% daily kcals from protein & $14.9(4)$ \\
\% daily kcals from fat & $39.5(7)$ \\
\% daily kcals from sweets & $15.4(15)$ \\
\% daily kcals from alcohol & $0(1.05)$ \\
Total MUFA, \% & $15.6(5.3)$ \\
Total PUFA, \% & $9.5(3.1)$ \\
Total SFA, \% & $11.0(2.8)$ \\
Total Trans, \% & $1.1(0.4)$ \\
Sodium, mg & $2156.3(1521)$ \\
\hline
\end{tabular}

Kcals $=$ calories, g=grams, MUFA = monounsaturated fatty acids, PUFA = polyunsaturated fatty acids, SFA = saturated fatty acids, Trans $=$ trans fatty acids.

Table 3. Alternate Healthy Eating Index-2010 total and component scores.

\begin{tabular}{|c|c|c|c|}
\hline & Intake for Max Score & $\begin{array}{l}\text { Point Range } \\
\text { (max) }\end{array}$ & $\begin{array}{c}\text { Sample } \\
\text { Median (IQR) }(n=126)\end{array}$ \\
\hline Whole Grains & Women $\geq 75 \mathrm{~g}$ & 0-10 (10) & $1.7(0.1,8.5)$ \\
\hline Total Fruit (not juice) & $\geq 4$ servings & $0-10(10)$ & $3.1(0.1,10)$ \\
\hline SSB and Fruit Juices & 0 servings & $0-10(10)$ & $1.04(0,10)$ \\
\hline Vegetables (excluding potatoes) & $\geq 5$ servings & $0-10(10)$ & $5.11(0.3,10)$ \\
\hline Nuts and Legumes & $\geq 1$ serving & $0-10(10)$ & $3.7(0.2,10)$ \\
\hline Red and Processed Meat & 0 servings & 0-10 (10) & $7.4(0,10)$ \\
\hline $\mathrm{EPA}+\mathrm{DHA}$ & $\geq 250$ milligrams & $0-10(10)$ & $3.0(0.03,10)$ \\
\hline PUFA & $\geq 10 \%$ of total energy intake & $0-10(10)$ & $9.3(2.6,10)$ \\
\hline Sodium & Lowest decile of sample population & $0-10(10)$ & $7.8(0,10)$ \\
\hline Alcohol (drinks/day) & Women $(0.5-1.5)$ & $0-10(10)$ & $5(0,10)$ \\
\hline Trans Fat & $\leq 0.5 \%$ of total energy intake & $0-10(10)$ & $8.3(3.1,10)$ \\
\hline $\begin{array}{l}\text { Total AHEI score } \\
(0-110 \text { points }) \text {, mean } \pm \text { SD }\end{array}$ & & & $57.4 \pm 10.9$ \\
\hline
\end{tabular}

$\mathrm{IQR}=$ interquartile range, AHEI = Alternate Healthy Eating Index-2010; $\max =$ maximum, $\mathrm{g}=$ grams, SSB = sugar sweetened beverages, PUFA = polyunsaturated fatty acids, EPA = eicosapentaenoic acid, DHA = docosahexaenoic acid, $\mathrm{SD}=$ standard deviation.

\subsection{Associations between Inflammation, Body Composition, OA Severity, and Diet Quality}

Results of correlational analysis revealed inverse associations between AHEI-2010 and BF\%, as well as for AHEI-2010 and BMI $(\rho=-0.20, p=0.03$ and $\rho=-0.21, p=0.02$, respectively). AHEI-2010 and IL-6 were inversely correlated $(\rho=-0.23, p=0.009)$. Positive correlations were found between BMI and IL-6 ( $\rho=0.19, p=0.03)$ as well as BMI and WOMACpf and $\rho=0.22, p=0.02)$. When adjusting for $\mathrm{BF} \%$ using partial correlations, there were no significant findings between AHEI-2010 and IL-6.

Results of unconditional logistic regression with IL-6 (high vs. low based on median split of $3.5 \mathrm{pg} / \mathrm{mL}$ ) as outcome variable revealed a significant inverse association for AHEI-2010 and IL-6 (Table 4). When WOMACpf was included in the model, the inverse association between AHEI-2010 and IL-6 remained ( $\beta=-0.04$, OR 0.96, CI 0.92-0.99), meaning those with higher DQ had $\sim 4 \%$ lower odds of having higher IL-6 based on median split and, as expected, WOMACpf $(\beta=0.04$, OR 1.04, 95\% CI 1.00-1.07) was also deemed a significant predictor of IL-6. Higher WOMACpf scores were associated with higher IL-6. VAT and BF\% were not significant predictors of inflammation. 
Table 4. Impact of diet quality (AHEI-2010), body composition, and osteoarthritis severity (WOMACpf) on high interleukin- 6 concentrations in females $(n=126)$.

\begin{tabular}{cccccc}
\hline Variables & Model 1 & Model 2 & Model 3 & Model 4 & Model 5 \\
\hline AHEI-2010 & $0.95(0.92-0.99) *$ & $0.96(0.92-0.99) *$ & $0.96(0.92-0.99) *$ & $0.95(0.92-0.99) *$ & $0.96(0.92-0.99)^{*}$ \\
VAT & - & $1.00(1.0-1.0)$ & $1.00(1.0-1.0)$ & - & - \\
BF\% & - & - & - & $1.00(0.93-1.07)$ & $0.96(0.93-1.07)$ \\
WOMACpf & - & - & $1.04(1.01-1.07) *$ & - & $1.04(1.01-1.07)^{*}$ \\
\hline
\end{tabular}

Data represented as odds ratio with (95\% confidence intervals). ${ }^{*}$ Significant $p$-value set at $<0.05$. IL- $6=$ interleukin 6 (median split at $\geq 3.5 \mathrm{pg} / \mathrm{mL}$ for high IL- 6 and $<3.5 \mathrm{pg} / \mathrm{mL}$ for low IL- 6 as outcome variable), VAT = visceral adipose tissue volume, AHEI-2010 $=$ Alternate Healthy Eating Index-2010, WOMACpf $=$ Western Ontario and McMaster Universities Osteoarthritis Index (osteoarthritis severity) physical function subcategory, BF\% = body fat percentage.

\section{Discussion}

Few studies have examined the association between DQ, body composition, OA severity and inflammation in persons with OA, particularly for AA older adults. The main finding of this study was that DQ, measured by AHEI-2010, and OA severity, measured by WOMAC, are significant predictors of systemic inflammation among older AA females with self-reported OA. A similar inverse association between DQ and inflammatory status has been reported by other researchers in various populations but not OA populations [27-31].

\subsection{Dietary Patterns and Quality of Diet}

In our cohort of older overweight and obese AA adults with self-reported OA, overall DQ was less than desirable since reported intakes were lower than nutritional guidelines. This is concerning given inverse associations have been found in the literature between AHEI-2010 score and chronic diseases like cardio-vascular disease (CVD), type 2 diabetes mellitus (T2DM) and chronic inflammation, which are generally more prevalent in AA individuals [6,32-34]. Comparing our results to two large prospective studies, researchers of the Nurses' Health Study (NHS) and the Health Professionals Follow-Up Study (HPFS) found that the highest AHEI-2010 scores (defined by $>57.8$ points for females in NHS cohort and $>62.3$ for males in HPFS) were negatively associated with major chronic disease risk, including CVD, T2DM, cancer, and non-traumatic death [25]. Other researchers using data from the National Institute of Health-American Association of Retired Persons (NIH-AARP) study (mean age $\sim 62$ years, primarily NHW) found that highest quintile AHEI-2010 scores (60.7-90.7 for females and 60.5-92.1 for males) were associated with lower risk of all-cause, cancer, and CVD mortality among females and males [35]. Another study examining DQ trends of a nationally representative sample ( $n=29,124, \sim 20 \%$ AA, 52\% female, 34\% within 60-85 years old), using AHEI-2010 to score DQ, found AA adults had lower AHEI-2010 scores than Mexican-American and NHW groups [36]. However, these differences in DQ did not remain between AA and NHW groups after adjusting for education and income [36]. These findings collectively suggest that DQ is lower for AAs and possibly affected by socioeconomic factors.

\subsection{Associations between Diet Quality (DQ) and Body Composition}

In support of our hypothesis, we found a significant inverse correlation between AHEI-2010 and $\mathrm{BF} \%$ for females but not between AHEI-2010 and VAT. Using a longitudinal study design, Maskarinec et al. [37] explored the associations of $\mathrm{DQ}$ in middle age on $\mathrm{BF} \%$ and VAT changes in older adulthood among a subgroup of participants from the Multi-ethnic cohort study (60-72 years at recruitment, $17 \%$ AA). They found that both male and female participants in the highest tertile of four DQ scores (HEI-2010, AHEI-2010, alternate MED (aMED), and Dietary Approaches to Stop Hypertension (DASH)) at baseline had lower total body fat at follow-up (mean time $20.9 \pm 1.2$ years) [37]. Participants in the higher tertile of the four DQ indices, including AHEI-2010 at baseline were also less likely to have high VAT at follow-up (HEI-2010 OR: 0.48, 95\% CI: 0.35, 0.66; AHEI-2010 OR: 0.62, 95\% CI: 0.46, 0.85; aMED 
OR: $0.65,95 \%$ CI: $0.47,0.90$; DASH OR: $0.41,95 \%$ CI: $0.30,0.58)$ [37]. This study provides supporting evidence that DQ can have inverse relationships with both $\mathrm{BF} \%$ and VAT for both sexes.

\subsection{Associations between Diet Quality and Inflammation}

In support of our stated hypothesis, we found inverse correlations between DQ and inflammation, more specifically AHEI-2010 and IL-6, in overweight and obese females with OA. However, no correlation between DQ and IL-6 existed in females when $\mathrm{BF} \%$ was adjusted for using partial correlation, suggesting that $\mathrm{BF} \%$ is an important contributor to inflammation. The correlational observations between AHEI-2010 and IL-6 from our study in AA females align with findings from other studies in various race/ethnic populations. Researchers using a subsample of adult women from the NHS cohort ( $n=660,100 \%$ female, 43-69 years old) found that of the five DQ indices used (original Healthy Eating Index, original AHEI, Recommended Food Score, Diet Quality Index-Revised, and aMED), AHEI had the strongest inverse associations with inflammatory markers after adjusting for age, BMI, and total kcal intake [27]. Study participants with the highest quintile of AHEI score had $31 \%$ lower IL-6 concentrations compared to those in the lowest quintile [27].

\subsection{Relationships between Diet Quality, Body Composition, Inflammation, and OA Severity}

We found notable relationships between $\mathrm{DQ}$, body composition, and OA severity on inflammatory markers in females. Results of our simple logistic regression model suggest that a higher AHEI-2010 score is attributed to a $\sim 4 \%$ higher odds (OR $0.953,95 \%$ CI $0.920-0.986, p=0.006$ ) of having "low" IL-6 $(<3.5 \mathrm{pg} / \mathrm{mL})$ in females. This adds to the emerging evidence that adherence to prudent dietary patterns may decrease chronic inflammation, and as our study demonstrates, even in populations prone to a higher inflammatory status as in OA. For example, Fung et al. [27] found higher adherence to AHEI-2010 was inversely associated with IL-6 values among females (43-69 years old) in the NHS cohort after adjusting for various demographic variables, physical activity, total kcal intake, and BMI. Overall, existing evidence, including findings from the present study, suggests that higher adherence to various "healthy" dietary patterns can positively impact inflammatory levels among various populations controlling for demographic characteristics (i.e., race, income, total kcal intake), physical activity, and body composition.

Although consistent with emerging evidence, results from our logistic regression model exploring $\mathrm{DQ}$, body composition, and OA severity were modest at best (only predicting $\sim 16 \%$, at most, of the variation in inflammation). This means other factors, known and unknown, which we did not account for could be contributing to inflammation. Collectively, our findings and existing evidence suggest that certain dietary changes to improve DQ may help reduce IL-6 levels in older adults with OA but findings may not be generalizable to all racial groups. Future studies with larger sample sizes are needed to substantiate these findings.

\subsection{Limitations and Strengths}

This study was not without limitations. We used a convenience sample of AA female adults, and our sample size precluded in-depth explorations of the inter-relationships between DQ, body composition, and inflammation. Our study is also limited by the use of only one inflammatory biomarker. We acknowledge that a comparison group consisting of other racial/ethnic groups might have allowed us to better interpret our findings. The cross-sectional design of this study made cause and effect relationships indeterminant. In addition, we scored dietary patterns based on data collected using FFQs which are prone to measurement and reporting errors. Lastly, our findings are only generalizable to urban, overweight and obese, older, AA females with OA.

Despite limitations as listed above, our study had many strengths. Results of our study help fill a gap in the literature related to overweight and obese older AA females with OA and provide descriptive DQ (AHEI-2010) data for this population. In addition, we explored relationships between DQ, body composition, and inflammation which are largely understudied in older AA adults. Data for 
the study was obtained by trained research personnel using validated measures (i.e., modified Block FFQ, DXA, DQ scores, anthropometric) and standardized protocols.

\section{Conclusions}

In conclusion, AHEI-2010 and WOMACpf were independent predictors of IL-6 suggesting that higher AHEI-2010 scores were related to lower IL-6 taking OA severity into account. Our study contributes to the existing literature by showing that DQ, as defined by AHEI-2010, accounted for $\sim 16 \%$ of the variation of IL-6 (inflammation) among older AA adult females with OA. Our findings suggest that higher DQ might help suppress inflammation in older AA females with OA. Further research is needed to explore the additional known and unknown factors (mediators and confounders) contributing to inflammation in AA older adults with OA.

Author Contributions: Conceptualization, M.M., L.-T.H., C.T., L.C., S.G.-P.; Methodology, M.M., S.L.H., M.F., C.T., L.-T.H., L.C., S.G.-P.; Validation, L.-T.H., C.T., S.G.-P.; Formal Analysis, M.M., S.G.-P.; Investigation, M.M., S.L.H., M.F.,L.-T.H, S.G.-P.; Resources, S.L.H., M.F., L.-T.H., S.G.-P.; Data Curation, L.-T.H.; Writing-Original Draft Preparation, M.M., S.G.-P.; Writing-Review and Editing, M.M., S.G.-P., C.T., L.-T.H., L.C., S.L.H., M.F.; Visualization, M.M., S.G.-P.; Supervision, S.G.-P.; Project Administration, L.-T.H., S.G.-P.; Funding Acquisition, M.F., S.H., L.-T.H.

Funding: This research was supported by R01AG039374 from the National Institute on Aging (S.H., M.F.), the American Cancer Society of Illinois grant \#2617755 (L.-T.H.) and American Cancer Society Mentored Research Scholar grant MRSG014-025-01-CNE (L.-T.H.).

Acknowledgments: The authors thank Andrew DeMott, P.J. Desai, Amy Shah, and Sarah Olender for their research and administrative support. We would also like to thank the study participants for giving their time generously to the project.

Conflicts of Interest: The authors declare no conflict of interest.

\section{References}

1. Lawrence, R.C.; Felson, D.T.; Helmick, C.G.; Arnold, L.M.; Choi, H.; Deyo, R.A.; Gabriel, S.; Hirsch, R.; Hochberg, M.C.; Hunder, G.G.; et al. National Arthritis Data Workgroup. Estimates of the prevalence of arthritis and other rheumatic conditions in the united states. Part II. Arthritis Rheum. 2008, 58, $26-35$. [CrossRef]

2. Robinson, W.H.; Lepus, C.M.; Wang, Q.; Raghu, H.; Mao, R.; Lindstrom, T.M.; Sokolove, J. Low-grade inflammation as a key mediator of the pathogenesis of osteoarthritis. Rheumatology 2016, 12, 580-592. [CrossRef] [PubMed]

3. Barbour, K.E.; Helmick, C.G.; Boring, M.; Qin, J.; Pan, L.; Hootman, J.M. Obesity trends among adults with doctor-diagnosed arthritis-united states, 2009-2014. Arthritis Care Res. 2017, 69, 376-383. [CrossRef]

4. National Institutes of Health: National Institute on Aging. Osteoarthritis. Available online: https://www. nia.nih.gov/health/osteoarthritis (accessed on 12 June 2017).

5. Centers for Disease Control and Prevention (CDC). Trends in aging-united states and worldwide. Morb. Mortal. Wkly. Rep. 2003, 52, 101-104, 106.

6. Dillon, C.F.; Rasch, E.K.; Gu, Q.; Hirsch, R. Prevalence of knee osteoarthritis in the united states: Arthritis data from the third national health and nutrition examination survey 1991-94. J. Rheumatol. 2006, 33, 2271-2279. [PubMed]

7. Hootman, J.M. Osteoarthritis in elderly persons: Risks of exercise and exercise as therapy. Clin. J. Sport Med. 2010, 20, 223. [CrossRef]

8. Fransson, E.I.; Batty, G.D.; Tabak, A.G.; Brunner, E.J.; Kumari, M.; Shipley, M.J.; Singh-Manoux, A.; Kivimaki, M. Association between change in body composition and change in inflammatory markers: An 11-year follow-up in the whitehall II study. J. Clin. Endocrinol. Metab. 2010, 95, 5370-5374. [CrossRef]

9. Sowers, M.; Jannausch, M.; Stein, E.; Jamadar, D.; Hochberg, M.; Lachance, L. C-reactive protein as a biomarker of emergent osteoarthritis. Osteoarthr. Cartil. 2002, 10, 595-601. [CrossRef]

10. Muthuri, S.G.; Hui, M.; Doherty, M.; Zhang, W. What if we prevent obesity? risk reduction in knee osteoarthritis estimated through a meta-analysis of observational studies. Arthritis Care Res. 2011, 63, 982-990. [CrossRef] 
11. Martel-Pelletier, J.; Barr, A.J.; Cicuttini, F.M.; Conaghan, P.G.; Cooper, C.; Goldring, M.B.; Goldring, S.R.; Jones, G.; Teichtahl, A.J.; Pelletier, J.P. Osteoarthritis. Nat. Rev. Dis. Primers 2016, 2, 1-18. [CrossRef]

12. Sohn, D.H.; Sokolove, J.; Sharpe, O.; Erhart, J.C.; Chandra, P.E.; Lahey, L.J.; Lindstrom, T.M.; Hwang, I.; Boyer, K.A.; Andriacchi, T.P.; et al. Plasma proteins present in osteoarthritic synovial fluid can stimulate cytokine production via toll-like receptor 4. Arthritis Res. Ther. 2012, 14, 1-13. [CrossRef] [PubMed]

13. Bellamy, N.; Buchanan, W.W.; Goldsmith, C.H.; Campbell, J.; Stitt, L.W. Validation study of WOMAC: A health status instrument for measuring clinically important patient relevant outcomes to antirheumatic drug therapy in patients with osteoarthritis of the hip or knee. J. Rheumatol. 1988, 15, 1833-1840. [PubMed]

14. Cornier, M.A.; Despres, J.P.; Davis, N.; Grossniklaus, D.A.; Klein, S.; Lamarche, B.; Council on the Kidney in Cardiovascular Disease, and Stroke Council. Assessing adiposity: A scientific statement from the american heart association. Circulation 2011, 124, 1996-2019. [CrossRef] [PubMed]

15. McCullough, M.L.; Feskanich, D.; Stampfer, M.J.; Giovannucci, E.L.; Rimm, E.B.; Hu, F.B.; Spiegelman, D.; Hunter, D.J.; Colditz, G.A.; Willett, W.C. Diet quality and major chronic disease risk in men and women: Moving toward improved dietary guidance. Am. J. Clin. Nutr. 2002, 76, 1261-1271. [CrossRef] [PubMed]

16. Wirt, A.; Collins, C.E. Diet quality-What is it and what does it matter? Public Health Nutr. 2009, 12, 2473-2492. [CrossRef]

17. Smith-Ray, R.L.; Fitzgibbon, M.L.; Tussing-Humphreys, L.; Schiffer, L.; Shah, A.; Huber, G.M.; Braunschweig, C.; Campbell, R.T.; Hughes, S.L. Fit and Strong! Plus: Design of a comparative effectiveness evaluation of a weight management program for older adults with osteoarthritis. Contemp. Clin. Trials 2014, 37, 178-188. [CrossRef]

18. Shamaileh, L.; Olender, S.; Castellanos, K.; Schiffer, L.; Welke, L.; Hughes, S.; Fitzgibbon, M.; Braunschweig, C.; Fantuzzi, G.; Tussing-Humphreys, L. Changes in Glucose Homeostasis and Systemic Inflammation Following an 8-week Exercise Only and 8-week Exercise Plus Dietary Weight Management Intervention among Overweight and Obese African American Older Adults with Osteoarthritis. Interdiscip. Undergrad. Res. J. 2016, 2, 14-25.

19. Centers for Disease Control and Prevention. Overweight and Obesity. Available online: https://www.cdc. gov/obesity/adult/defining.html (accessed on 23 August 2018).

20. Block, G. A review of validations of dietary assessment methods. Am. J. Epidemiol. 1982, 115, 492-505. [CrossRef]

21. Coates, R.J.; Eley, J.W.; Block, G.; Gunter, E.W.; Sowell, A.L.; Grossman, C.; Greenberg, R.S. An evaluation of a food frequency questionnaire for assessing dietary intake of specific carotenoids and vitamin $\mathrm{E}$ among low-income black women. Am. J. Epidemiol. 1991, 134, 658-671. [CrossRef]

22. Mares-Perlman, J.A.; Klein, B.E.; Klein, R.; Ritter, L.L.; Fisher, M.R.; Freudenheim, J.L. A diet history questionnaire ranks nutrient intakes in middle-aged and older men and women similarly to multiple food records. J. Nutr. 1993, 123, 489-501. [CrossRef]

23. Anderson, A.L.; Harris, T.B.; Tylavsky, F.A.; Perry, S.E.; Houston, D.K.; Lee, J.S.; Kanaya, A.M.; Sahyoun, N.R. Dietary patterns, insulin sensitivity and inflammation in older adults. Eur. J. Clin. Nutr. 2012, 66, 18-24. [CrossRef] [PubMed]

24. Kubo, A.; Block, G.; Quesenberry, C.P., Jr.; Buffler, P.; Corley, D.A. Dietary guideline adherence for gastroesophageal reflux disease. BMC Gastroenterol. 2014, 14, 1-9. [CrossRef] [PubMed]

25. Chiuve, S.E.; Fung, T.T.; Rimm, E.B.; Hu, F.B.; McCullough, M.L.; Wang, M.; Stampfer, M.J.; Willett, W.C. Alternative dietary indices both strongly predict risk of chronic disease. J. Nutr. 2012, 142, 1009-1018. [CrossRef] [PubMed]

26. Corty, E.W. Using and Interpreting Statistics: A Practical Text for the Health, Behavioral, and Social Sciences; Mosby/Elsevier: St. Louis, MO, USA, 2007.

27. Fung, T.T.; McCullough, M.L.; Newby, P.K.; Manson, J.E.; Meigs, J.B.; Rifai, N.; Willett, W.C.; Hu, F.B. Diet-quality scores and plasma concentrations of markers of inflammation and endothelial dysfunction. Am. J. Clin. Nutr. 2005, 82, 163-173. [CrossRef] [PubMed]

28. Hermsdorff, H.H.; Volp, A.C.; Puchau, B.; Barbosa, K.B.; Zulet, M.A.; Bressan, J.; Martinez, J.A. Contribution of gender and body fat distribution to inflammatory marker concentrations in apparently healthy young adults. Inflamm. Res. 2012, 61, 427-435. [CrossRef] [PubMed] 
29. Ko, B.J.; Park, K.H.; Shin, S.; Zaichenko, L.; Davis, C.R.; Crowell, J.A.; Joung, H.; Mantzoros, C.S. Diet quality and diet patterns in relation to circulating cardiometabolic biomarkers. Clin. Nutr. 2016, 35, 484-490. [CrossRef] [PubMed]

30. Mattei, J.; Sotres-Alvarez, D.; Gellman, M.; Castaneda, S.F.; Hu, F.B.; Tucker, K.L.; Siega-Riz, A.M.; Kaplan, R.C. Diet quality, inflammation, and the ankle brachial index in adults with or without cardiometabolic conditions. Clin. Nutr. 2018, 37, 1332-1339. [CrossRef]

31. Schwingshackl, L.; Hoffmann, G. Mediterranean dietary pattern, inflammation and endothelial function: A systematic review and meta-analysis of intervention trials. Nutr. Metab. Cardiovasc. Dis. 2014, 24, 929-939. [CrossRef]

32. Ma, M.; Ma, A. Racial/Ethnic differences in knowledge of personal and target levels of cardiovascular health indicators. J. Community Health 2015, 40, 1024-1030. [CrossRef]

33. Onvani, S.; Haghighatdoost, F.; Surkan, P.J.; Larijani, B.; Azadbakht, L. Adherence to the healthy eating index and alternative healthy eating index dietary patterns and mortality from all causes, cardiovascular disease and cancer: A meta-analysis of observational studies. J. Hum. Nutr. Diet. 2016, 1-11. [CrossRef]

34. Gerst-Emerson, K.; Jayawardhana, J. Loneliness as a public health issue: The impact of loneliness on health care utilization among older adults. Am. J. Public Health 2015, 105, 1013-1019. [CrossRef] [PubMed]

35. Reedy, J.; Krebs-Smith, S.M.; Miller, P.E.; Liese, A.D.; Kahle, L.L.; Park, Y.; Subar, A.F. Higher diet quality is associated with decreased risk of all-cause, cardiovascular disease, and cancer mortality among older adults. J. Nutr. 2014, 144, 881-889. [CrossRef] [PubMed]

36. Wang, D.D.; Leung, C.W.; Li, Y.; Ding, E.L.; Chiuve, S.E.; Hu, F.B.; Willett, W.C. Trends in dietary quality among adults in the united states, 1999 through 2010. JAMA Intern. Med. 2014, 174, 1587-1595. [CrossRef] [PubMed]

37. Maskarinec, G.; Lim, U.; Jacobs, S.; Monroe, K.R.; Ernst, T.; Buchthal, S.D.; Shepherd, J.A.; Wilkens, L.R.; Le Marchand, L.; Boushey, C.J. Diet quality in midadulthood predicts visceral adiposity and liver fatness in older ages: The multiethnic cohort study. Obesity 2017, 25, 1442-1450. [CrossRef] [PubMed]

(C) 2018 by the authors. Licensee MDPI, Basel, Switzerland. This article is an open access article distributed under the terms and conditions of the Creative Commons Attribution (CC BY) license (http:/ / creativecommons.org/licenses/by/4.0/). 